\title{
Obesidade e sua influência sobre o câncer: uma recente revisão da literatura
}

\author{
Obesity and its influence on cancer: a recent literature review \\ Carolina Freitas ${ }^{1}$ \\ ttps://orcid.org/0000-0002-2071-2895 \\ Raquel Alves dos Santos ${ }^{3}$ \\ Orcid: https://orcid.org/0000-0002-0075-2099 \\ Jaqueline Lopes Damasceno ${ }^{2}$ \\ Orcid: https://orcid.org/0000-0001-6852-6103 \\ Marina Garcia Manochio-Pina ${ }^{4}$ \\ Orcid: https://orcid.org/0000-0002-7851-050
}

\begin{abstract}
Resumo
Introdução: As taxas de obesidade têm aumentado em todo o mundo, sendo atualmente essa condição reconhecida como a principal causa evitável de vários tipos de câncer. Objetivo: Portanto, procurou-se realizar um levantamento das características clínicas que relacionam a obesidade e os diferentes tipos de câncer, entre os anos de 2017 e 2020. Materiais e Métodos: Revisão integrativa da literatura, com buscas de evidências nas bases de dados PubMed, Scielo e LILACS, cuja questão norteadora foi: "Qual a sobre a influência da obesidade em relação aos diferentes tipos de câncer?”. Resultados e Conclusões: Foram identificados 32 artigos, dos quais 8 foram selecionados. A gordura corporal foi enfatizada como um fator de grande impacto no desenvolvimento de neoplasias. Os dados sugerem que quatro em 10 tipos de câncer são evitáveis por meio de mudanças no comportamento e padrão alimentar do indivíduo. Estudos epidemiológicos têm mostrado uma forte ligação entre o fator dietético e a incidência e prognóstico do câncer. Considerando que a dieta e o exercício físico são fatores modificáveis, os projetos e as intervenções nos cuidados primários são essenciais para a prevenção da doença. $\mathrm{O}$ entendimento dos mecanismos biológicos explica como um comportamento saudável promove melhorias, destacando-se a importância da disseminação da informação às populações de risco e na implementação de estratégias que ajudem os pacientes a fazer escolhas melhores e mais saudáveis.
\end{abstract}

Palavras-chave: obesidade; neoplasias; alimentação; atividade física

\begin{abstract}
Introduction: Obesity rates have increased worldwide, and this condition is currently recognized as the main preventable cause of various types of cancer. Objective: Therefore, an attempt was made to survey of clinical characteristics that relates obesity and different types of cancer, between the years 2017 and 2020. Materials and Methods: Integrative literature review, with searches for analysis in the PubMed, Scielo and LILACS databases, specific guiding question was: "What is the influence of obesity in relation to different types of cancer?". Results and Conclusions: 32 articles were indentified, and then 8 were selected. Body fat was emphasized as a factor of important impact in the development of neoplasia. The data suggest that four of 10 types of cancer are preventable through changes in the individual's behavior and eating pattern. Epidemiological studies have shown a strong link between the dietary factor and the incidence and prognosis of cancer. Considering that diet and exercise are modifiable factors, primary care projects and interventions are essential for disease prevention.
\end{abstract}

\footnotetext{
${ }^{1}$ Universidade de Franca, SP, Brasil. E-mail: carolfreitas.nut@gmail.com

${ }^{2}$ Universidade de Franca, SP, Brasil. E-mail: jaquelinelopes.bio@hotmail.com

${ }^{3}$ Universidade de Franca, SP, Brasil. E-mail: raquel.santos@unifran.edu.br

${ }^{4}$ Universidade de Franca, SP, Brasil. E-mail: marina.manochio@unifran.edu.br
} 
Understanding the biological mechanisms explains how healthy behavior promotes improvements, highlighting the importance of disseminating information to risk populations and implementing strategies that help patients make better and healthier choices.

Keywords: obesity; neoplasia; feed; physical activity

\section{Introdução}

As taxas de obesidade têm aumentado em todo mundo, sendo essa condição reconhecida como uma das principais causas evitáveis de vários tipos de câncer $^{1,2}$. Várias doenças estão diretamente relacionadas com a obesidade, incluindo diabetes, hipertensão, aterosclerose, acidentes vasculares, doenças musculoesqueléticas e câncer, de modo que a obesidade é um problema prevalente na saúde pública $^{3}$. A ligação entre uma elevada adiposidade e o aumento do risco ao câncer são biologicamente plausíveis considerando que a obesidade está relacionada a uma vasta alteração metabólica e disfunções fisiológicas. As anormalidades na resistência insulínica e no sistema insulin growth fator (IGF-1), via da insulina, podem desencadear $\mathrm{o}$ desenvolvimento tumoral ${ }^{4}$. Conforme Pearson-Stuttard et $a l^{5}$ o diabetes e o elevado valor de índice de massa corporal (IMC) têm sido associados com o aumento de vários tipos de câncer, sugerindo que $3,9 \%$ da incidência global dos casos de câncer em 2012 foram atribuídos a valores elevados de IMC.

Recentemente, a literatura traz que o risco e a mortalidade por cânceres de tireoide, esôfago, fígado, vesícula biliar, cólon e rim, bem como linfoma não Hodgkin e mieloma múltiplo, estão particularmente associados à obesidade em ambos os sexos. Essa tendência também é forte para câncer de mama endometrial e pós-menopausa em mulheres e para câncer de próstata em homens ${ }^{6}$. Estima-se que mais de 14 milhões de pessoas no mundo desenvolvem câncer todo ano e esse número poderá aumentar para mais de 21 milhões até $2030^{7}$. Adicionalmente, 19,3 milhões de novos casos (18,1 milhões excluindo câncer de pele não melanoma) e quase 10,0 milhões de mortes ocorreram em 2020 no mundo todo ${ }^{8}$. No Brasil, considerado um dos países com maiores números de mortalidade no mundo e ocupando o segundo lugar em casos de óbito pela doença ${ }^{9}$, em 2018 , houve 559.371 novos casos, com 243.588 mortes. De acordo com a incidência, os cinco principais tipos de câncer são mama, próstata, pulmão, cólon e tireoide, sendo o câncer de pulmão, mama, próstata, estômago e cólon responsáveis pela maioria das mortes ${ }^{10}$.

O IMC elevado está associado a um aumento de 14 tipos de câncer. Um estudo calculou a incidência de câncer atribuído ao IMC elevado no Brasil no ano de 2012, e suas projeções para 2025. Foi encontrado que $3,8 \%$ de todos os cânceres diagnosticados em 2012 foram atribuídos ao IMC elevado, com maior incidência em mulheres $(5,2 \%)$ do que em homens $(2,6 \%)$. As populações que apresentaram maior incidência para todos os tipos de câncer foram nos estados mais ricos do país, localizados no sul e sudeste. Projeta-se que em 2025, os casos de câncer atribuídos a um elevado IMC no Brasil poderão atingir $4,6 \% \quad(6,2 \%$ em mulheres e $3,2 \%$ nos homens $)^{11}$.

De acordo com o World Cancer Research Fund International e o American Institute for Cancer Research, aproximadamente um terço das neoplasias mais comuns poderia ser evitada pela mudança do estilo de vida e hábitos alimentares nos países desenvolvidos ${ }^{12}$. Recentemente, estudos sugerem que a inflamação local e sistêmica causada pela adiposidade possui um papel central no câncer de mama, sendo assim, necessária uma maior atenção para populações de risco como, por exemplo, pacientes com 
hiperinsulinemia e/ou inflamação do tecido adiposo ${ }^{5}$.

A obesidade está associada com o aumento dos receptores positivos de estrogênio pós-menopausa, acrescendo o risco do câncer de mama e a relação dos subtipos de tumores mamários. Vários mecanismos têm contribuído para a ligação entre a obesidade e esse tipo de câncer, incluindo altos níveis de estrogênio local e circulante, elevação das adipocinas (lepitina e adiponectina), rompimento da sinalização da insulina/IGF, modificações no microbioma, e efeitos inflamatórios locais e sistêmicos ${ }^{5}$. Dados confirmam que o aumento da informação sobre o conceito do IMC e obesidade, e o crescimento do risco de cânceres ginecológicos que se relacionam com o excesso de adiposidade poderiam promover uma melhor conscientização dos riscos entre as mulheres ${ }^{13}$.

O aumento da ocidentalização associa-se aos fatores de risco para o aumento de câncer. De acordo com o Food and Agriculture Organization (FAO) os alimentos industrializados têm influenciado a cultura alimentar que antes era baseada em alimentos in natura e minimamente processadas ${ }^{10}$. Durante as últimas décadas, muitos países aumentaram drasticamente o consumo de alimentos ultraprocessados. Depois de submetidos a vários processos físicos, biológicos e/ou processos químicos, esses alimentos são processados para serem microbiologicamente seguros, altamente palatáveis e de preço acessível.

Os alimentos ultraprocessados podem conter alto teor de gorduras totais, gordura saturada e adição de açúcares e sal, junto com o baixo teor de fibras e vitaminas. Além da composição nutricional, esses alimentos podem conter propriedades carcinogênicas (pela presença de acrilamidas, aminas heterocíclicas, e hidrocarbonetos aromáticos policíclicos), podendo estar presentes durante o processo de aquecimento como resultado na reação de Maillard ${ }^{12}$. Em um estudo europeu foi avaliada a associação dos indicadores antropométricos da obesidade, o fator de risco estabelecido para vários tipos de câncer, distribuição da gordura corporal, que reflete nas complicações metabólicas da obesidade, e a relação da obesidade total e local, com a incidência de câncer. A circunferência de quadril, e a razão cintura quadril mostraram uma associação positiva com a relação obesidade e câncer, destacando-se o câncer colorretal em idosos ${ }^{4}$.

De acordo com Fiolet et al. ${ }^{12}$ um estudo realizado em 2009 na França, evidenciou que o consumo de alimentos ultraprocessados está associado a um aumento de risco de câncer em geral e de câncer de mama. Esses resultados mantiveram-se estatisticamente significativos após um ajuste de vários marcadores nutricionais. Um aumento de $10 \%$ no consumo de alimentos ultraprocessados está associado a um aumento de $12 \%$ nos riscos de cânceres em geral e $11 \%$ no risco de câncer de mama.

O estudo de Tabung et al. ${ }^{14}$ analisou a relação das dietas pró-inflamatórias com o aumento do risco de câncer colorretal, baseados nos níveis de biomarcadores inflamatórios circulantes. Os resultados sugeriram que essa inflamação é um potencial mecanismo ligado aos padrões alimentares e o desenvolvimento desse tipo de câncer. Intervenções para reduzir o efeito adverso das dietas pró-inflamatórias podem ser mais efetivas entre homens com obesidade ou sobrepeso ou mulheres, ou homens magros que não consumam álcool.

Além da alimentação sabe-se que exercícios físicos possuem um fator protetor contra o câncer colorretal, mesmo em indivíduos com diferentes IMC. As taxas de mortalidade por câncer colorretal no Brasil, entre 1990 e 2015, foram maiores que no restante do mundo $(68.47 \%$ e $24.56 \%$, respectivamente). A inatividade física foi responsável por 1.302 mortes em 1990 e 4.143 em 2015, devido ao câncer colorretal. Os estados brasileiros com melhores condições socioeconômicas indicaram maiores taxas de mortalidade e 
morbidade por câncer colorretal devido à inatividade física ${ }^{15}$. Obesidade, diabetes e câncer são doenças metabólicas. Ambos os tipos de diabetes 1 e 2 compartilham com o câncer mudanças hormonais (alterações na insulina IGF-1 e dos eixos lepitina/adiponectina), metabólicas, e da imunidade (aumentando a circulação de citocinas inflamatórias) ${ }^{16}$.

A obesidade tem influência de 20 a $35 \%$ de todos os cânceres. Os principais candidatos relacionados à obesidade são aquelas citocinas que causam resistência insulínica: lepitina, fator de necrose tumoral $\alpha(\mathrm{TNF}-\alpha)$, adiponectina e ácidos graxos livres. A resistência insulínica e a hiperinsulinemia promovem uma produção de crescimento de fator 1 insulin-like (IGF1). Várias linhagens de células cancerígenas, incluindo de cólon e próstata, têm receptores IGF-1. Os adipócitos viscerais, pela via da lipólise, aumentam a circulação dos ácidos graxos livres que podem apresentar um potencial cancerígeno, através da proliferação celular, da estimulação do IGF-1, e indiretamente, através da resistência insulínica ${ }^{17}$.

Existem outros mecanismos pelos quais a glicose favorece o crescimento tumoral, independentemente da insulina: a indução do aumento no crescimento de fatores como IGF nas células cancerígenas; aumento celular invasivo e migratório; aumento das espécies reativas de oxigênio e produtos glicosilados. As células tumorais obtêm energia através da fosforilação oxidativa e com a alta disponibilidade de glicose a produção de espécies reativas de oxigênio é aumentada, favorecendo o desenvolvimento de mutações ${ }^{17}$.

De acordo com o que foi discutido acima, é sugerido que a hiperglicemia seja um fator de risco independente dos fatores tumorais e inflamatórios para o desenvolvimento do câncer em pacientes diabéticos. Recentemente, os resultados de pesquisa em câncer têm enfatizado a importância da imunidade, metabolismo e dos oncometabólitos. $\mathrm{O}$ conceito emergente é de que certos metabólitos modulam a sinalização nas células tumorais, induzindo as adaptações requeridas para suportar todas as características tumorais ${ }^{16}$.

Evitar o ganho de peso através da restrição calórica pode melhorar a inflamação no tecido adiposo branco em ratos. A perda de peso está associada com a redução da interleucina 5 (IL-5), fator de estimulação de colônias de granulócitos humanos recombinantes (GM-CSF), e neutrófilos circulantes, resultando na reversão dos efeitos pró-metastáticos da obesidade em modelos de câncer de mama pré-clínicos. Intervenções no estilo de vida como atividade física, têm apresentado melhoras no sistema imune. Os exercícios físicos e a epinefrina levam à ativação das células T-killer de imunovigilância, e suprimem a incidência tumoral e o crescimento de vários tipos de câncer ${ }^{18}$.

Dentro desse contexto, o objetivo deste estudo foi realizar um levantamento das características clínicas que relacionam a obesidade e os diferentes tipos de câncer, entre os anos de 2017 e 2020.

\section{Materiais e Métodos}

Este estudo constitui-se de uma revisão integrativa da literatura, cuja questão norteadora foi: "Qual a influência da obesidade em relação aos diferentes tipos de câncer?".

Para o critério de seleção dos artigos científicos foram utilizados os seguintes descritores de saúde (DeCS): "obesidade", "excesso de peso corporal", "câncer" e "alimentos processados", na base de dados US National Library of Medicine National Institutes of Health (PubMed) Scientific Electronic Library Online (SciELO), Literatura Latino-Americana e do Caribe em Ciências da Saúde (LILACS), no mês de abril de 2020. Foram selecionados artigos científicos em português, inglês e espanhol.

Como critério de inclusão os artigos publicados entre os anos de 2017 e 2020, devido à relevância do tema na atualidade. Foram excluídos os estudos divulgados em outros formatos que não fossem artigos (por 
exemplo: dissertações, teses, livros, capítulos de livro, editoriais, entre outros), aqueles publicados em outro idioma que não o mencionado anteriormente, bem como aqueles que não estavam de acordo com o tema. Esses critérios de exclusão foram considerados na análise dos títulos e resumos dos estudos e, posteriormente, na leitura dos textos recuperados na íntegra.

Como limitações do estudo, foram encontrados poucos artigos dentro do período proposto que abordassem diretamente o tema, ainda, percebeu-se que a literatura recente é escassa em estudos que relacionem a obesidade a tipos específicos de cânceres, principalmente em questão a publicações em português.

\section{Resultados}

Após a consulta nas bases de dados anteriormente mencionadas, foram identificados 32 artigos, sendo: PubMed $(n=30)$, LILACS $(n=2)$. Quando aplicados os critérios de exclusão apenas 8 artigos foram selecionados, os quais corresponderam aos critérios metodológicos.

A Figura 1 apresenta o fluxograma do processo de seleção dos artigos pesquisados. As informações mais relevantes dos trabalhos foram apresentadas na Quadro 1. 
Freitas C, Damasceno JP, Santos RA, Manochio-Pina MG

Figura 1: Fluxograma do processo de seleção dos artigos pesquisados. Brasil, 2020.

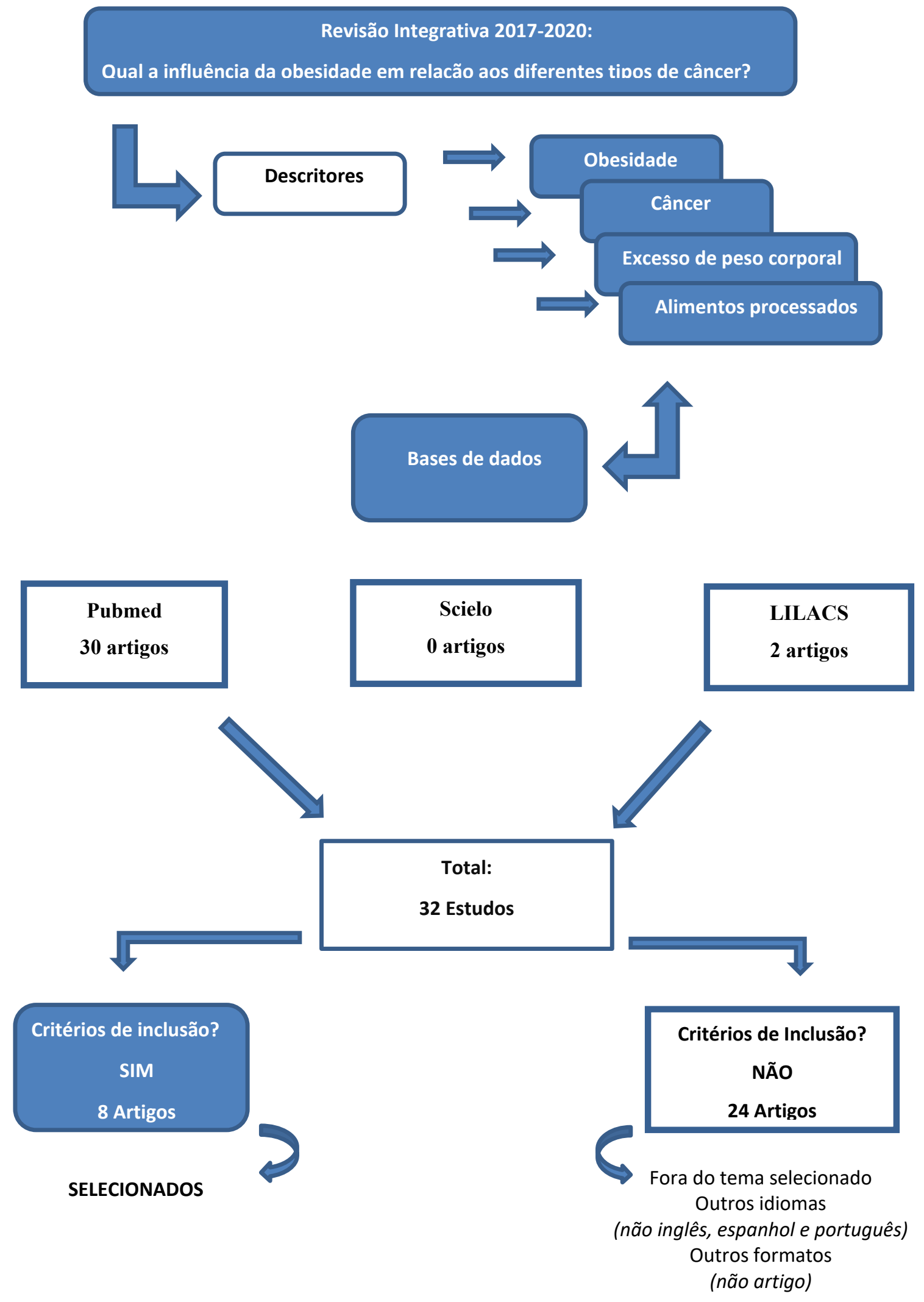

Fonte: Elaborada pelos autores 
Quadro 1. Resultados da análise dos artigos. Brasil, 2020.

\begin{tabular}{|c|c|}
\hline ARTIGO & DESCRIÇÃO DOS DADOS \\
\hline Autor / Ano & Stern et al. $^{27}$ \\
\hline Título & $\begin{array}{l}\text { Dieta, atividade física, obesidade os riscos relacionados ao câncer: estratégias para reduzir } \\
\text { a sobrecarga de câncer nas Américas }\end{array}$ \\
\hline Objetivo & $\begin{array}{l}\text { Discutir as relações entre dieta, obesidade, atividade física e câncer, enfatizando } \\
\text { estratégias direcionadas às crianças para diminuir o risco de obesidade, controlar fatores } \\
\text { de risco relacionados à obesidade e reduzir estilos de vida sedentários. }\end{array}$ \\
\hline Conclusão & $\begin{array}{l}\text { Os vínculos entre dieta, obesidade, atividade física e câncer, enfatizando estratégias } \\
\text { direcionadas às crianças para diminuir o risco de obesidade, controlar fatores de risco } \\
\text { relacionados à obesidade e reduzir o estilo de vida sedentário, considerando que esses } \\
\text { fatores poderá ter alto impacto no risco de câncer em adultos. }\end{array}$ \\
\hline Autor / Ano & Aguirre-Portolés \& Molina ${ }^{19}$ \\
\hline & A nutrição precisa para combater o metabolismo lipídico no câncer colorretal \\
\hline Objetivo & $\begin{array}{l}\text { Aprofundar o estudo de eventos coocorrentes, que orquestram a tumorogênese da CRC e } \\
\text { são essenciais para a evolução paradigmas nutricionais de precisão. Discutir a aplicação } \\
\text { de nutrição de precisão abordagens para atingir o metabolismo lipídico na CRC. }\end{array}$ \\
\hline Conclusão & $\begin{array}{l}\text { Evidências convincentes obtidas de estudos epidemiológicos e experimentais apoiam o } \\
\text { papel crucial da obesidade, padrões alimentares, interações gene-dieta e metabolismo } \\
\text { lipídico na prevenção e prognóstico da CRC. O desenho de ensaios clínicos que combinam } \\
\text { agentes quimioterapêuticos clássicos com produtos bioativos visando o metabolismo } \\
\text { lipídico constitui uma linha inquestionável de pesquisa no tratamento de CRC. }\end{array}$ \\
\hline Autor / Ano & Yang et al..$^{29}$ \\
\hline Título & $\begin{array}{l}\text { Fatores de risco de câncer para jovens e adultos: obesidade, inflamação e mecanismos } \\
\text { sociocomportamentais. }\end{array}$ \\
\hline Objetivo & $\begin{array}{l}\text { O artigo avalia as disparidades sociais sobre os riscos metabólicos e inflamatórios do } \\
\text { câncer na população jovem e adulta dos EUA, e examina os mecanismos psicossociais e } \\
\text { comportamentais nessas disparidades. }\end{array}$ \\
\hline Conclusão & $\begin{array}{l}\text { Este estudo fornece novos conhecimentos sobre a distribuição social de exposições } \\
\text { precoces da vida a precedentes fisiológicos ao desenvolvimento do câncer mais tarde na } \\
\text { vida, com implicações para a prevenção e intervenção precoce de comportamentos de risco } \\
\text { modificáveis em adolescentes e adultos jovens. }\end{array}$ \\
\hline Autor / Ano & López-Suárez ${ }^{25}$ \\
\hline Título & Casos de câncer atribuível à obesidade, diabetes tipo 2 e fatores de risco associados. \\
\hline Objetivo & $\begin{array}{l}\text { Discorrer como a obesidade desempenha um papel central na morbidade e mortalidade de } \\
\text { doenças de múltiplos órgãos e sistemas e é um dos principais contribuintes para a crescente } \\
\text { incidência de câncer. }\end{array}$ \\
\hline Conclusão & $\begin{array}{l}\text { Aproximadamente } 30 \text { a } 40 \% \text { dos cânceres mais comuns são evitáveis, evitando o fumo e } \\
\text { o excesso de peso, adotando um estilo de vida saudável. Ter um estilo de vida saudável } \\
\text { significa praticar atividade física regular; escolher uma dieta predominantemente baseada } \\
\text { em vegetais que limite o consumo de carne vermelha, álcool e alimentos ultraprocessados, } \\
\text { particularmente carne vermelha processada; e selecionar legumes, nozes, frutas secas e } \\
\text { peixe como a principal fonte de proteínas. No entanto, também são solicitadas } \\
\text { intervenções de saúde pública na entrega de alimentos para reduzir o ônus dos cânceres } \\
\text { relacionados à obesidade e para aliviar as crescentes despesas insustentáveis para os } \\
\text { sistemas de saúde. }\end{array}$ \\
\hline
\end{tabular}


Continuação

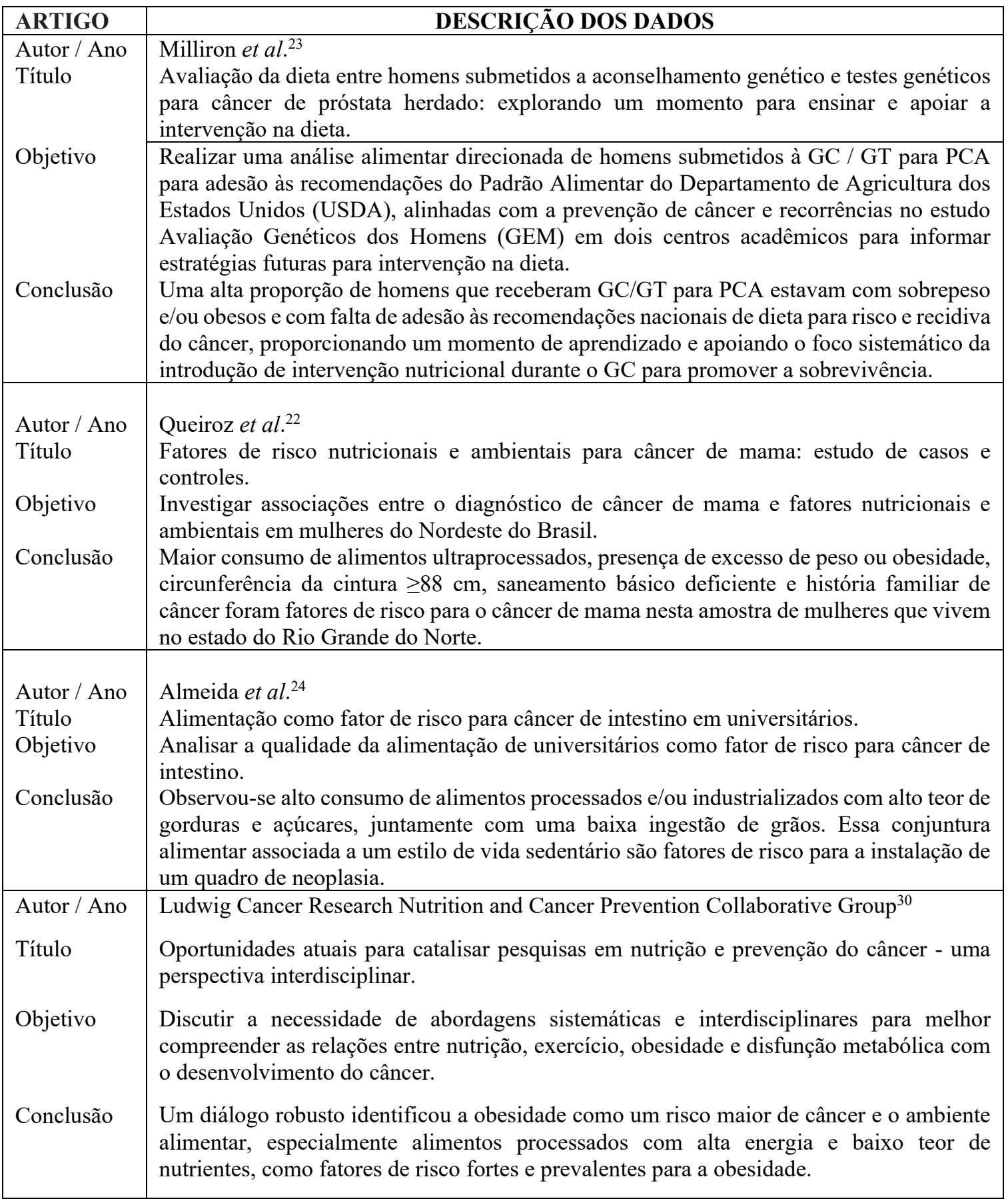

De acordo com os artigos encontrados, todos enfatizam a gordura corporal como um fator de grande impacto no desenvolvimento de neoplasias. Os dados sugerem que quatro em 10 tipos de câncer são evitáveis por meio de mudanças no comportamento do indivíduo, principalmente em relação ao padrão alimentar, assim como evitar o comportamento sedentário, destacando a nutrição e a atividade física como os principais fatores de prevenção do câncer. 


\section{Discussão}

Durante a última década, a diminuição da atividade física e o aumento da ingestão calórica contribuíram para o aumento da obesidade, dessa forma ocorreu uma mudança metabólica no organismo, dando origem a hipertrofia e hiperplasia do tecido adiposo ${ }^{19}$. A suscetibilidade ao câncer não depende somente dos antecedentes genéticos do paciente, os fatores ambientais e o estilo de vida podem determinar a etiologia do câncer colorretal. Apesar dos avanços de diagnóstico precoce e terapias específicas para esse tipo de câncer, a incidência tem aumentado em pessoas com menos de 40 anos de idade. O consumo de álcool, fumo, disbiose, dietas ricas em carne vermelha, estão fortemente associadas ao câncer colorretal ${ }^{14}$.

Em consonância com o estudo citado anteriormente, encontrado nesta revisão, conforme Silva et al. ${ }^{15}$ o câncer colorretal é considerado o terceiro tipo mais letal de câncer em mulheres e o quarto em homens no Brasil, enquanto nos Estados Unidos representa o segundo nessa classificação. Essa neoplasia tem uma etiologia multifatorial que inclui genética e fatores de estilo de vida modificáveis. Entre os fatores de estilo de vida modificáveis, a atividade física se destaca, pois ela interage com alguns genes que são capazes de influenciar o início do desenvolvimento desse câncer. Contudo, evidências mostram que a prática de atividade física regular pode reduzir o desenvolvimento do câncer colorretal entre $20-25 \%$ em ambos os sexos. Esses mecanismos ainda não estão claros, mas mudanças nas funções imunes e mudança nos níveis de prostaglandinas, insulina, secreção da bile, colesterol e hormônios gastrointestinais têm sido relatados.

Segundo Freitas-Alves et al. ${ }^{20} \mathrm{o}$ câncer de mama é o mais frequente entre as mulheres, e sua crescente incidência é um desafio mundial. A exposição ao estrogênio é o principal fator de risco, porém a obesidade pós-menopausa tem se mostrado um gatilho para o desenvolvimento e progressão da doença. A ligação entre a obesidade e a carcinogênese do câncer de mama envolve o aumento da produção de estrogênio e estímulos pró-inflamatórios no tecido adiposo, com ativação da via da ciclooxigenase-2. Nesse estudo foi avaliado o impacto dos polimorfismos da ciclogenase-2 em combinação com a obesidade, no risco da progressão do câncer de mama nos pacientes brasileiros. Pôde-se concluir que a genotipagem da ciclogenase2 pode ser adicionada como avaliação prognóstica em pacientes obesas com câncer de mama. De acordo com um estudo $^{21}$ que avaliou os fatores de risco para o câncer de mama na população no sudeste do Brasil, 4.242 mulheres, entre 40 e 69 anos sem o histórico de câncer de mama, foram avalidas e o modelo de predição de risco indicou que as variáveis podem ser avaliadas na seguinte população: idade, altura, biópsia mamária prévia, número de gravidez, uso de terapia de reposição hormonal.

Outro estudo brasileiro $^{22}$ identificado nesta revisão investigou associações de fatores de risco para o câncer de mama. O saneamento básico deficiente das moradias e histórico familiar de câncer foi significativamente associado. De acordo com os dados antropométricos, o excesso de peso e a circunferência de cintura aumentada apresentaram uma prevalência maior no grupo de casos diagnosticados, o consumo regular de alimentos ultraprocessados também foi identificado como um fator de risco para câncer de mama.

O baixo consumo de frutas, vegetais e frutos do mar, e o elevado consumo de carnes processadas e alimentos ricos em gorduras saturadas, foram relatados entre homens diagnosticados com câncer de próstata não metastásico. A adesão a uma dieta Mediterrânea, rica em vegetais e ômega 3 , tem sido associada a 
baixos ínidices de motaliadde relacionada a esse tipo de câncer ${ }^{23}$.Alguns fatores semelhantes sobre a conduta alimentar têm relação com o aumento do risco do câncer colorretal, a obesidade e o alto consumo de carnes vermelhas, de gorduras e de álcool. No entanto, uma dieta equilibrada, rica em fibras, vitaminas e minerais, pode ser fator preventivo, e pode auxiliar juntamente ao tratamento clínico e no retrocesso do estágio carcinogênico ${ }^{24}$.

$\mathrm{Na}$ obesidade, especificamente no diabetes tipo 2, há um estado de regulação positiva nos receptores IGF, hiperinsulinemia, resistência à insulina e dislipidemia, ambas condições estão associadas à um estado inflamatório que aumenta os níveis de interleucina 6 (IL-6), TNF- $\alpha$, e inibidor do ativador plasminogênio. Essas alterações ativam os receptores celulares levando às mudanças complexas nas vias de sinalização, e acredita-se que essas mudanças têm um papel crítico no risco de oncogênese, através da proliferação e migração, angiogênese, e diminuição da apoptose celular $^{25}$.

Resultados similares demonstraram que o aspecto fisiopatológico da obesidade e do diabetes é a inflamação crônica caracterizada pelo aumento da produção das citocinas pró-inflamatórias (IL-6, TNF- $\alpha$ ) pelo tecido adiposo, o que leva à resistência insulínica. Altos níveis de IL-6 e TNF- $\alpha$ têm sido reportados em pacientes com diferentes tipos de câncer. A sinalização das citocinas inflamatórias por meio das proteínas quinases, como proteinocinases ativadas por mitógenos (MAPK), ou da família Janus de tirosinoquinases/fatores de transcrição (JAK/STAT), contribui para a biologia do câncer através do aumento da proliferação, sobrevivência, e acumulação de mutações nos tumores celulares ${ }^{16}$.

Ainda, Guitierrez-Salmeron et al. ${ }^{16}$ trazem que sistematicamente, esses fatores contribuem para supressão da imunidade antitumoral do organismo. Os altos níveis circulantes de glicose promovem o crescimento tumoral através de mecanismos diretos e indiretos. A captação de glicose é aumentada nas células tumorais devido às adaptações metabólicas que elas sofreram. A glicose induz indiretamente $o$ crescimento tumoral através do aumento dos níveis circulantes de fatores de crescimento (insulina/IGF-1), fator de crescimento epidermal (EGF) e citocinas inflamatórias.

Segundo Stern et al. ${ }^{26}$ o processo de globalização é destacado como um grande influenciador de alguns fatores de risco, considerando que crianças e adultos estão expostos a esses fatores independente do país de residência. A população latina que vive nos Estados Unidos está experimentando uma transição dietética, que antes era composta de grãos, legumes, frutas e vegetais, e agora repleta de alimentos processados, de alta densidade calórica, e bebidas altamente adoçadas. Essa transição também é caracterizada por comportamentos sedentários, obesidade, doenças cardiovasculares, diabetes e câncer.

O aumento da compreensão desses processos metabólicos pode gerar intervenções efetivas com o intuito de reduzir a incidência global da obesidade e consequentemente a incidência de câncer. De acordo com o aumento da prevalência desses fatores de risco para o câncer, os esforços globais e guias clínicos deveriam refletir sobre a importância do câncer como uma consequência da diabetes e IMC elevado, podendo assim direcionar medidas preventivas a fim de reduzir a morbidade nesse grupo de pacientes ${ }^{3}$. Essa combinação do padrão alimentar, atividade física, peso corporal, e inflamação podem explicar os $35 \%$ dos cânceres em adultos atribuíveis às dietas e má nutrição ${ }^{27}$.

Uma dieta balanceada e diversificada pode ser considerada um dos fatores modificáveis mais importantes na prevenção dos riscos primários do câncer ${ }^{12}$. Rezende et al. ${ }^{11}$ ressaltam a importância das intervenções na saúde pública e nas políticas (políticas fiscais e regulamentação de rotulagem, marketing e venda de 
produtos ultra processados), as quais são necessárias para redução dos níveis de sobrepeso e obesidade da população. Concomitante, políticas de guias alimentares e inovações de mercado devem valorizar e promover alimentos frescos e minimamente processados, promovendo dessa forma o desenvolvimento de sistemas alimentares saudáveis, sendo apropriados culturalmente e viáveis economicamente no Brasil.

Yang et $a .^{28}$ relatam que a obesidade e o excesso de gordura corporal associados à dieta ocidental podem representar a maior causa evitável de câncer de não fumantes. A inatividade física pode aumentar ainda mais o risco de vários tipos de câncer através dos seus efeitos na adiposidade, além da inativação do sistema imune e inflamação.

O baixo teor de gordura corporal é protetor contra alguns cânceres. No entanto, é necessário compreender como uma dieta específica e processos metabólitos interagem com fatores ambientais. Contudo, fatores dietéticos e metabólicos possuem um papel na prevenção do câncer, apoiado por uma base científica já elucidada até o momento ${ }^{29}$.

Ações para combater a inatividade física, educação alimentar e ações em políticas públicas a fim de melhorar a classificação do câncer e tratamento são urgentes nos estados brasileiros ${ }^{25}$. A promoção de modos de vida ativos requer uma integração de vários setores da saúde para aumentar o número de oportunidades a fim de encorajar indivíduos para a atividade física. Intervenções políticas e ambientais (ciclovias, calçadas apropriadas para caminhada e parques) são essenciais para apoiar mudanças sustentáveis em atividade física em grande parte da população ${ }^{10}$.

A Organização Mundial da Saúde e a Sociedade Americana de Câncer recomendam aos pacientes que sobreviveram ao câncer consumirem uma dieta com base em vegetais e realizarem 150 minutos por semana de atividade física com intensidade moderada. As modificações no estilo de vida têm mostrado benefícios na prevenção do câncer, redução dos seus riscos e fatores que favorecem a qualidade de vida ${ }^{30}$.

\section{Conclusão}

De acordo com os dados recentes aqui apresentados, indicadores antropométricos ligados à obesidade, elevado IMC e acúmulo de gordura corporal geram disfunções metabólicas e fisiológicas que podem favorecer uma maior incidência de diferentes tipos cancerosos. Portanto, o presente estudo contribui para um melhor entendimento dos mecanismos biológicos que ligam obesidade e câncer, entre eles, processos inflamatórios, alterações na microbiota e hormonais, anormalidades no sistema imunológico bem como relacionadas à resistência insulínica, os quais estão entre as modificações clínicas atualmente relatadas na literatura.

Considerando que a dieta e o exercício físico são fatores modificáveis na prevenção de vários tipos de câncer, os projetos e as intervenções nos cuidados primários são essenciais para a prevenção da doença. Um comportamento saudável promove melhorias, de modo que a importância da disseminação da informação às populações de risco e a implementação de estratégias que ajudem os pacientes a fazer escolhas saudáveis, que possam ser sustentadas de acordo com seu estilo de vida, requerem o engajamento em um conjunto de ações intersetoriais para que as estratégias de mobilização que visam incentivar e apoiar ambientes de alimentação adequada e saudável sejam atingidas. 


\section{Referências}

1. World Health Organization; Obesity: preventing and managing the global epidemic

2. Report of a WHO Consultation (WHO Technical Report Series 894), 2000.

3. World Cancer Research Foundation. American Institute for Cancer Research. Food, nutrition, physical activity and the prevention of cancer: a global perspective. Washington: American Institute for Cancer Research, 2007.

4. Argolo DF, Hudis CA, Iyengar NM. The Impact of obesity on breast cancer. Curr Oncol Rep Brazil. 2018; 20(6): 47.

5. Freisling H, Arnold M, Soerjomataram I, O'doherty MG, Ordóñez-Mena JM, et al. Comparison of general obesity and measures of body fat distribution in older adults in relation to cancer risk: meta-analysis of individual participant data of seven prospective cohorts in Europe. Br J Cancer. 2017; 116(11): 1486-1497.

6. Pearson-Stuttard J, Zhou B, Kontis V, Bentham J, Gunter MJ, et al. Worldwide burden of cancer attributable to diabetes and high body-mass index: a comparative risk assessment. Lancet Diabetes Endocrinol. 2018; 6(2): 95-104.

7. Ackerman SE, Blackburn OA, Marchildon F, Cohen P. Insights into the link between obesity and cancer. Curr Obes Rep. 2017(6): 195-203.

8. Siegel, RL, Miller, KD. Jemal, A. (2015). Cancer statistics, 2015. CA: a câncer Journal for clinicians, 65(1), 5-29.

9. Sung, H, Ferlay, J, Siegel, RL, Laversanne, M, Soerjomataram, I, Jemal, A, \& Bray, F. (2021). Global cancer statistics 2020: GLOBOCAN estimates of incidence and mortality worldwide for 36 cancers in 185 countries. CA: A Cancer Journal for Clinicians).

10. Carvalho DDNR, De Aguiar VFF, Neresmartins JD, Bendelaque DDFR, Sardinha DM, et al. Cancer mortality in the north region of Brazil in the historical series. 2010-2017. IJAERS. 2020; 7(3): 174-181.

11. Oliveira AF, Quadros CA, de Castro Ribeiro HS, Wainstein AJA, de Queiroz Sarmento, et al. (2021). Global Forum of Cancer Surgeons: Support for the Brazilian Society of Surgical Oncology Journey towards Implementation of Cytoreductive Surgery/Hyperthermic Intraperitoneal Chemotherapy in Brazil. Annals of Surgical Oncology).

12. Rezende LFM, Arnold M, Rabacow FM, Levy RB, Claro RM, et al. The increasing burden of cancer attributable to high body mass index in. Cancer Epidemiol. 2018; 28; 54:63-70.

13. Fiolet T, Srour B, Sellem L, Kesse-Guyot E, Allès B, et al. Consumption of ultraprocessed foods and cancer risk: results from NutriNet-Santé prospective cohort. BMJ. 2018; 14; 360: k322.

14. Armbruster S, Solimen PT. Public knowledge of obesity and gynecologic cancer risk. In: Berger N, Klopp A, Lu K. (eds) Focus on gynecologic malignancies. Energy Balance and Cancer. 2018; 13. Springer, Cham.

15. Tabung FK, Liu L, Wang W, Fung TT, Wu K, et al. Association of dietary inflammatory potential with colorectal cancer risk in men and women. JAMA Oncol. 2018; 4(3): 366373.

16. Silva DAS, Tremblay MS, Souza MDFMD, Mooney M, Naghavi M, et al. Mortality and years of life lost by colorectal cancer attributable to physical inactivity in Brazil (19902015): Findings from the Global Burden of Disease Study. PLoS One. 2018; 13(2): e0190943. 
17. Guitierrez-Salmeron M, Chocarro-Calvo A, García-Martinez JM, De La Vieja A, GarciaGimenez C. Epidemiological bases and molecular mechanisms linking obesity, diabetes, and cancer. Endocrinol Diabetes Nutr. 2017; 64(2): 109-117.

18. Akbari ME, Gholamalizadeh M, Doaei S, Mirsafa F. FTO gene affects obesity and breast cancer through similar mechanisms: a new insight into the molecular therapeutic targets. Nutr Cancer. 2018; 70(1): 30-36.

19. Olson OC, Quail DF, Joyce JA. Obesity and the tumor microenvironment. Science. 2017; 358(6367): 1130-1131.

20. Aguirre-Portoléz C, Fernandéz L, Molina A. Precision nutrition for targeting lipid metabolism in colorectal cancer. Nutrients. 2017; 9(10): 1076.

20. Freitas-Alves DR, De Andrade Vieira-Monteiro H, Piranda DN, Sobral-Leite M, Da Silva TSL, et al. PTGS2 polymorphism rs689466 favors breast cancer recurrence in obese patients. Endocr Relat Cancer. 2018; 25(3): 351-365.

21. Breyer JZ, Wendland EM, Kops NL, Caleffi M, Hammes LS. Assessment of potential risk factors for breast cancer in a population in southern Brazil. Breast Cancer Res Treat. 2018; 169(1): 125-131.

22. Queiroz de Sousa IM, De Melo Silva FR, De Oliveira Lyra C, Fayh PT. Nutritional and environmental risk factors for breast cancer: a case-control study. Sci med. 2018; 28(2): ID28723.

23. Milliron BJ, Bruneau M, Obeid E, Gross L, Bealin L, et al. Diet assessment among men undergoing genetic counseling and genetic testing for inherited prostate cancer: Exploring a teachable moment to support diet intervention. Prostate. 2019; 79(7): 778-783.

24. Almeida L, Santos BT, Prates RP, Leão LL, Pereira JV, et al. Alimentação como fator de risco para câncer de intestino em universitários. RBPS. 2017; 30(1): 72-78.

24. Lopez-Suaréz AL. Burden of cancer attributable to obesity, type 2 diabetes and associated risk factors. Metabolism. 2019; 92: 136-146.

25. Stern MC. Diet, physical activity, obesity and related cancer risk: strategies to reduce cancer burden in the Americas. Salud Publica Mex. 2019; 61(4): 448-455.

26. Giovannucci E A. framework to understand diet, physical activity, body weight, and cancer risk. Cancer Causes Control. 2018; 29: 1-6.

27. Yang YC, Johnson MP, Schorpp KM, Boen CE, Harris KM. Young adult risk factors for cancer: Obesity, inflammation, and sociobehavioral mechanisms. Am J Prev Med. 2017; 53(3S1): S21-S29.

28. Ludwig - Cancer Research Nutrition and Cancer Prevention Collaborative Group - UK. Cancer research nutrition and cancer prevention collaborative group. Current opportunities to catalyze research in nutrition and cancer prevention - an interdisciplinary perspective. BMC Med. 2019; 17(1): 148.

29. Mehra K, Berkowitz A, Sanft T. Diet, physical activity, and body weight in cancer survivorship. Med Clin North Am. 2017; 101(6): 1151-1165.

Como citar este artigo:

Freitas C, Damasceno JP, Santos RA, Manochio-Pina MG. Obesidade e sua influência sobre o câncer: uma recente revisão da literatura. Rev. Aten. Saúde. 2021; 19(67): 344-356. 